\title{
Processing scarce biological samples for light and transmission electron microscopy
}

P. Taupin ${ }^{1,2,3}$

\author{
${ }^{1}$ National Neuroscience Institute; ${ }^{2}$ National University of Singapore; ${ }^{3}$ Nanyang Technological University \\ 11 Jalan Tan Tock Seng, Singapore
}

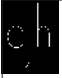

(C)2008 European Journal of Histochemistry

Light microscopy (LM) and transmission electron microscopy (TEM) aim at understanding the relationship structure-function. With advances in biology, isolation and purification of scarce populations of cells or subcellular structures may not lead to enough biological material, for processing for LM and TEM. A protocol for preparation of scarce biological samples is presented. It is based on pre-embedding the biological samples, suspensions or pellets, in bovine serum albumin (BSA) and bis-acrylamide (BA), cross-linked and polymerized. This preparation provides a simple and reproducible technique to process biological materials, present in limited quantities that can not be amplified, for light and transmission electron microscopy.

Key words: araldite, bis-acrylamide, bovine serum albumin, cross-link, cryostat, embedding, LR White.

Correspondence: Philippe Taupin,

11 Jalan Tan Tock Seng, Singapore

Tel.: +65.6357 - 7533 .

Fax: +65.6256 - 9178 .

E-mail: obgpjt@nus.edu.sg

Paper accepted on April 27, 2008

European Journal of Histochemistry

2008; vol. 52 issue 2 (Apr-Jun): 135-139

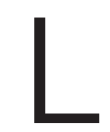

ight and transmission electron microscopy are powerful techniques for studying the relationship structure-function of organisms, tissues and cells (Leapman 2004). They offer incomparable levels of resolution and sensitivity, for studying biological samples. However, they are suited for samples for which critical amounts of material can be obtained, through isolation and purification, or amplification. With advances in biology, it is possible to isolate and purify scarce biological samples; organisms, cells or subcellular structures. When preparation of such samples can not be scaled-up or amplified, the limited amount of material available may prevent its processing, for LM and TEM (Zhao et al. 2004).

Protocols routinely used to process biological samples available in limited quantities, for LM and TEM, involve agarose or gelatin pre-embedment. However, these procedures have inherent limitations; among them, the difficulty in identifying the samples in the solidified gel and the fragility of the gel. To overcome these limitations, we report a simple and efficient preparation to preembed scarce biological samples for LM and TEM. The ability to efficiently and reproducibly process biological samples available in limited quantity, for microscopy techniques, will contribute to advances in cell biology.

\section{Material and Methods}

\section{Cell culture}

For this study, 3T3 fibroblasts were cultured, according to standard protocols. Briefly, cells were passaged with trypsin, harvested by centrifugation (800 rpm, $3 \mathrm{~min}$ ) and resuspended in phosphate buffer saline (PBS). Cells were counted in a hematocytometer. Aliquots of 1,000-3,000 cells were fixed in $4 \%$ paraformaldehyde (PF) and 
$0.25 \%$ glutaraldehyde (GA), in PBS, at RT for $10 \mathrm{~min}$. Cells were then pelleted in a bench top centrifuge, at full speed, at RT for $3 \mathrm{~min}$. The pellets -barely visible- were either resuspended in 23.1 $\mu \mathrm{L}$ PBS or kept as pellets. In this latter case, the supernatants were carefully removed. The cells, as suspensions or pellets, were embedded in BSA and BA, as described below.

\section{Embedding matrix}

The following mix or embedding matrix was prepared aside for each samples, by mixing: 23.1 $\mu L$ of PBS - only for samples kept as pellet (Gibco), $6.6 \mu \mathrm{L}$ of $30 \%$ BSA (Gibco), $5 \mu \mathrm{L}$ of $40 \%$ BA (BioRad) and $1 \mu L$ of Temed (BioRad) (Table 1). Note, when processing samples, as pellet, the embedding matrix must be carefully layered on the top of the pellet, so that it maintains its integrity.

\section{Cross-linking and polymerizing mix}

To cross-link and polymerize the embedding matrix - containing the samples, the following mix, or cross-linking and polymerizing mix, was prepared aside and immediately before use: 24 $\mu \mathrm{L}$ of $12.5 \% \mathrm{PF}$ were mixed with $2 \mu \mathrm{L}$ of $25 \%$ $\mathrm{GA}$ and $5 \mu \mathrm{L}$ of $10 \%$ ammonium persulfate. 6.2 $\mu \mathrm{L}$ of the cross-linking and polymerizing mix were added to the embedding matrix, and gently mixed. Alternatively, the embedding matrix, without the samples, and the cross-linking and polymerizing mix can be mixed independently, and added to the samples. The final volume for each sample (pellet or suspension) is $41.9 \mu \mathrm{L}$. The final concentrations of $B S A, B A, P F$ and $G A$ are $4.7 \%, 4.8 \%, 1.43 \%$ and $0.24 \%$, respectively.

The samples were left at RT for 1 to 1 h 30 min, for polymerization of the embedding matrix. After polymerization, the embedding matrix has the appearance of a gel-like substance. The samples were then transferred in PBS.

Table 1. Embedding matrix.

\begin{tabular}{lcc}
\hline & Pellet & Suspension resuspended in 23.1 mL PBS \\
\hline $30 \% \mathrm{BSA}$ & $23.1 \mu \mathrm{L}$ & - \\
$40 \% \mathrm{BA}$ & $6.6 \mu \mathrm{L}$ & $6.6 \mu \mathrm{L}$ \\
Temed & $5 \mu \mathrm{L}$ & $5 \mu \mathrm{L}$ \\
\hline
\end{tabular}

PBS, phosphate buffer saline; BSA, bovine serum albumin; BA, bis-acrylamide.

\section{Light microscopy}

For LM, the biological samples, embedded in the matrix, were transferred in sucrose $30 \%$, in phosphate buffer and left at $4^{\circ} \mathrm{C}, 0 / \mathrm{N}$. The tissue samples were then embedded in OCT-tissue tek (OCT compound, Miles Inc) and frozen in isopentane, for 5 min. The blocks were sectioned on a cryostat. Twenty $\mu \mathrm{m}$ sections were cut out and layered on Superfrost/Plus glass slides. The glass slides were stained with cresyl violet and examined on a light microscope.

\section{Transmission electron microscopy}

For TEM, the gel-like samples were sliced into 1 $\times 2 \times 2 \mathrm{~mm}$ pieces. When processing the pellets, the gel-like samples were observed under a light microscope to visualize and cut out the pieces containing the biological material. Two types of resin were assayed: araldite and LR White (Electron Microscopy Sciences). For embedding in araldite, the pre-embedded samples were submitted to the following process of dehydration, infiltration and embedding: water at RT for 20 min, twice; 50\% ethanol at RT for 10 min, once; $75 \%$ ethanol at RT for $20 \mathrm{~min}$, once; 95\% ethanol at RT for $20 \mathrm{~min}$, once; $100 \%$ ethanol at RT for 20 min, once; $100 \%$ acetone at RT for 20 min, twice; araldite:acetone (1:1) at RT for $30 \mathrm{~min}$, once; araldite:acetone (6:1) at RT O/N; pure araldite at RT for $20 \mathrm{~min}$, then at $40^{\circ} \mathrm{C}$ for $30 \mathrm{~min}$; pure araldite at $45^{\circ} \mathrm{C}$ for $\mathrm{l} \mathrm{h}$ and pure araldite at $50^{\circ}$ for $1 \mathrm{~h}$. Finally, the samples were transferred in gelatin capsules and let polymerize in pure araldite, at $60^{\circ} \mathrm{C}$ for $48 \mathrm{~h}$.

For LR White embedding, the samples were submitted to the following process: water at RT for 20 min, twice; $50 \%$ ethanol at RT for 10 min, once; $75 \%$ ethanol at RT for 20 min, once; $95 \%$ ethanol at RT for 20 min, once; $100 \%$ ethanol at RT for 20 min, three times; LR White:ethanol (1:6) at RT for $4 \mathrm{~h}$, once; pure LR White at RT O/N; pure LR White at RT for $1 \mathrm{~h}$, twice; pure LR White at RT O/N and pure LR White at $50^{\circ} \mathrm{C}$ for $1 \mathrm{~h}$, three times. Finally, the samples were transferred in gelatin capsules, sealed and let polymerize in pure LR White, at $50^{\circ} \mathrm{C}$ for 3-4 days.

\section{Semi-thin and thin sections}

Semi-thin and thin sections were prepared using an ultramicrotome (Leica). Thin sections were mounted on formvar-coated grid. The grids were stained with uranyl acetate, at RT for $10 \mathrm{~min}$, fol- 
lowed by lead citrate, at RT for 10 min. Grids were examined on a JEOL JSM 1010 electron microscope, at $100 \mathrm{kV}$, using the Gatan software with the Dual vision 300W camera model 780 (NUS electron microscopy department).

\section{Results and Discussion}

A preparative technique is reported to process scarce biological samples, samples present in limited quantities that can not be amplified, for LM and TEM. The protocol is based on pre-embedding the biological material in a matrix of BSA and BA, cross-linked and polymerized. The matrix is compatible with most LM and TEM protocols and procedures.

Under light microscope, sparse densities of cells were observed on cryostat sections of fibroblasts, pre-embedded as suspension, and stained with cresyl violet (Figure la). Pictures taken at high magnification reveal that the morphology of the cells is well preserved (Figure 1b). Similarly, aggregates of cells were observed on cryostat sections of fibroblasts pre-embedded as pellet (data not shown). Under electron microscope, aggregates of cells were observed on thin sections of fibroblasts pre-embedded as pellet, in LR White, at low magnification (Figure 1c). Pictures taken at high magnification from these sections reveal that the morphology of the cells and cellular organelles, like mitochondria and smooth endoplasmic reticulum, are well preserved (Figure 1d-e). Similarly, aggregates of cells were observed on thin sections of fibroblasts preembedded as pellet, in araldite, and their morphology were also well preserved (Figure If). When samples of fibroblasts pre-embedded as suspension, in LR White or araldite, were analyzed, single cells were occasionally observed (data not shown). However, the quality of the electron microscopy data is affected by the fact that the samples were not stained with osmium after their post-fixation, in PF and GA. Osmium post-fixation is a usual procedure for conventional ultrastructural morphology. This step can not be performed for scarce biological samples pre-embedded in BSA and BA, for two main reasons. First, the scarcity of the samples studied prevents osmium post-fixation, before their pre-embedment in BSA and BA. This would result in samples contaminated with osmium or the loss of the samples, with the subsequent washes required to wash away the osmium. Second, once preembedded in BSA and BA, the matrix becomes dark as a result of the staining with osmium (unpublished data). This disturbs both the detection and observation of the samples (unpublished data). Despite this, these results show that the preparation of the samples, pre-embedded in BSA and BA, is compatible with standard LM and TEM procedures.
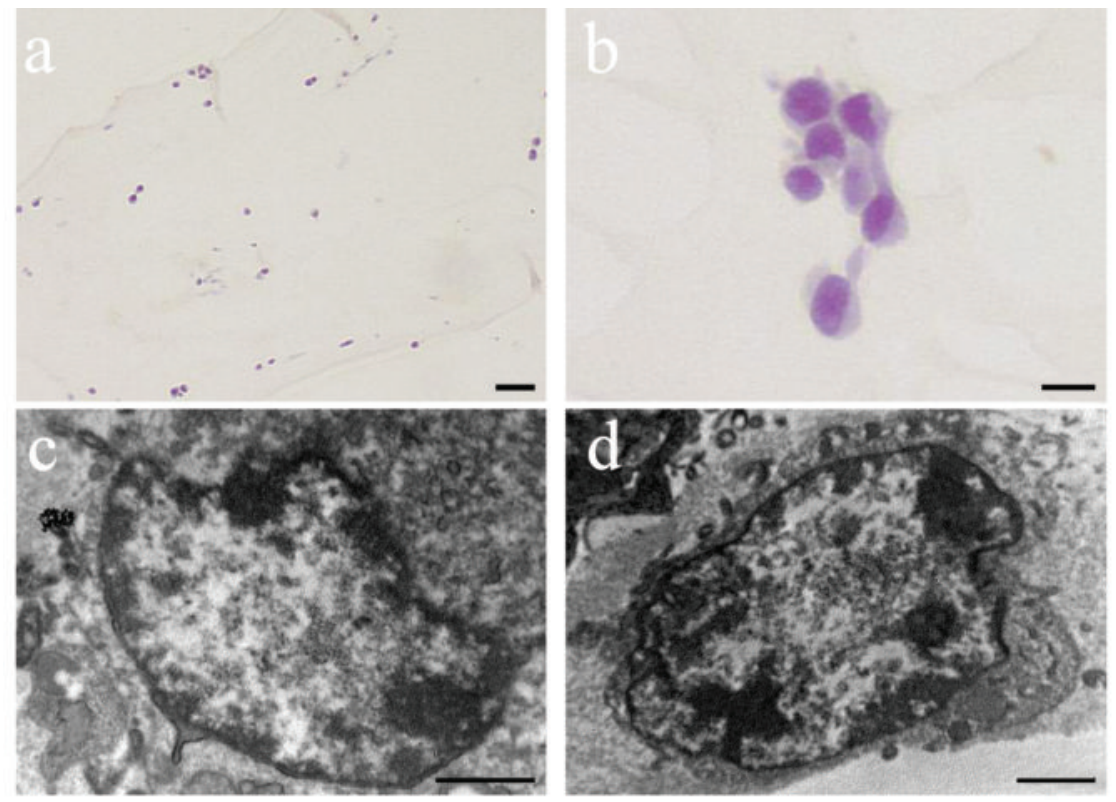

Figure 1. Photografhs and electron micrographs of fibroblasts pre-embedded in bovine serum albumin and bis-acrylamide. (a) Low magnification of a representative field of a cryostat section containing fibroblasts, pre-embedded as suspension. The fibroblasts, pre-embedded in BSA and BA, were embedded in OCTtissue tek, processed for histology and stained with cresyl violet. (b) higher magnification of a cryostat section containing fibroblasts, pre-embedded as suspension. c,d: fibroblasts pre-embedded as pellet in BSA and BA and processed for electron microscopy: the fibroblasts were embedded either in LR White (c) or araldite (d), and stained with uranyl acetate and lead citrate. Scale bar: a, 4 $\mu \mathrm{m} ; \mathrm{b}, 10 \mu \mathrm{m} ; \mathrm{c}$ and $\mathrm{d}, 1 \mu \mathrm{m}$. 
Preparing samples for LM and TEM typically requires many times more material than what is needed for observation, under the microscope. Quite often, however, one is faced with samples that are too scarce to be securely handled through all the standard washes, dehydration and infiltration fluid changes, involved in LM and TEM. Investigators have developed various ways to immobilize tiny samples, a clump of several cells for example. Among them, embedding in gelatin or agarose are procedures most commonly used (Malatesta et al. 1999). These have been mostly some shared through channels, like communications between technologists. Regarding the use of BSA, it has been previously reported for embedding biological material for freeze-fracture cytochemistry (Pinto da Silva et al. 1981). Tissue was embedded in $30 \%$ BSA, cross-linked with $1 \%$ $\mathrm{GA}$, at RT for $30 \mathrm{~min}$. We optimized the protocol for pre-embedding in BSA, for LM and TEM. We found that a mixture of $5 \% \mathrm{BSA}$ and $5 \% \mathrm{BA}$, cross-linked with PF and $G A$, and polymerized with ammonium persulfate, were optimal concentrations to produce a gel-like matrix, capable of pre-embedding cells and subcellular structures. As a result of lower concentrations of reagents used in this protocol, particularly BSA, a longer time ( 1 to $1 \mathrm{~h} 30 \mathrm{~min}$ ) is required for the matrix to polymerize. In this example, the cells have been fixed with PF $4 \%$ and GA $0.25 \%$, for $10 \mathrm{~min}$, before pre-embedding. Since the final concentrations of PF and GA during pre-embedding are $1.43 \%$ and $0.24 \%$, respectively, it is not necessary to fixe the tissue before embedding in BSA and $B A$, using this protocol. We report that the gel-like matrix is compatible with most standard LM and TEM protocols and procedures. For LM, it is compatible with cryostat and paraffin block (date not shown), preparation and sectioning. For TEM, it is compatible with both araldite and LR White resins; the translucence and viscosity of $L R$ White facilitating the handling of the samples pre-embedded in BSA and BA, throughout the process of dehydration, infiltration and embedding. LR White is used when investigators aim at performing post-embedding immunological reactions (Timms 1986). However, the conditions for performing such immunostaining, on samples preembedded in BSA and BA, remain to be established and validated.
The use of BSA coupled with BA offers several advantages and benefits for processing scarce biological samples, over other procedures. The gel-like matrix formed with BSA and BA is translucent, making it possible to reliably identify the cells in the polymerized gel and cut them out, as opposed to agarose or gelatin that are opaque once solidified. Further, the use of BA to polymerize the gel-like matrix offers a more resistant matrix for processing samples, throughout the histological procedures. It will particularly hold together the samples much better than either gelatin, agarose or BSA alone, particularly for TEM processing that requires multiple and longer steps. The pre-embedding procedure reported represents therefore an improvement over most commonly used ones.

Scarce biological samples may be processed as pellet or suspension (Rodriguez-Boulan et al. 1983; Sanz et al. 2003). Studying scarce biological samples, processed as pellet, under the microscope is less tedious than those processed as suspension, particularly for TEM. Because of the translucent properties of BSA and BA, the protocol reported here is particularly suited for processing biological samples, as pellet, as it may be visualized under the microscope once embedded in the matrix. This represents another advantage of the embedding procedure reported here. Data presented here reported the processing of 1,000 to 3,000 cells of starting material. In theory, this protocol could be applied with lower amount of starting material, up to single cells. However, analyzing lower density of cells may represent a challenging and tedious task.

In conclusion, a simple and efficient preparative technique is described that allows studying scarce biological samples for LM and TEM. The use of BSA and BA, for pre-embeding, offers several advantages for preparing scarce biological samples, over traditional processes commonly used, and is compatible with a broad array of microscopy procedures. This protocol is particularly suitable for collection of scarce isolated cells and rare human material.

\section{Acknowledgments}

I wish to thank Ms Janice Tan Meiying, Mrs Wai Lin Loh, Mr Lu Thong Beng and Ms Tan Suat Hoon for expert technical assistance. 


\section{References}

Leapman RD. Novel techniques in electron microscopy. Curr Opin Neurobiol 2004; 14:591-98.

Malatesta M, Mannello F, Sebastiani M, Bianchi G, Gazzanelli G. Prostate-specific antigen found in type I breast cyst fluids is a secretory product of the apocrine cells lining breast gross cysts. Breast Cancer Res Treat 1999; 57:157-63.

Pinto da Silva P, Torrisi MR, Kachar B. Freeze-fracture cytochemistry: localization of wheat-germ agglutinin and concanavalin A binding sites on freeze-fractured pancreatic cells. J Cell Biol 1981;91:36172.
Rodriguez-Boulan E, Paskiet KT, Sabatini DD. Assembly of enveloped viruses in Madin-Darby canine kidney cells: polarized budding from single attached cells and from clusters of cells in suspension. J Cell Biol 1983;96:866-74.

Sanz R, Battu S, Puignou L, Galceran MT, Cardot PJ. Sonication effect on cellular material in sedimentation and gravitational field flow fractionation. J Chromatogr A 2003;1002:145-54.

Timms BG. Postembedding immunogold labeling for electron microscopy using "LR White" resin. Am J Anat 1986;175:267-75.

Zhao X, Hilliard LR, Mechery SJ, Wang Y, Bagwe RP, Jin S, et al. A rapid bioassay for single bacterial cell quantitation using bioconjugated nanoparticles. Proc Natl Acad Sci USA 2004;101:1502732. 